\title{
Optical constants of beryllium thin layers determined from Mo/Be multilayers in spectral range 90 to $134 \mathrm{eV}$
}

\author{
Mewael Giday Sertsu $\odot,{ }^{\text {a,* Andrey Sokolov, }}{ }^{\text {a }}$ Nikolay Chkhalo, ${ }^{b}$ \\ Vladimir Polkovnikov, ${ }^{\mathrm{b}}$ Nikolay Salashchenko, ${ }^{\mathrm{b}}$ \\ Mikhail Svechnikov $\odot,{ }^{b}$ and Franz Schäfers ${ }^{\text {a }}$ \\ ${ }^{a}$ Helmholtz-Zentrum Berlin für Materialien und Energie, BESSY II, \\ Department Optics and Beamlines, Berlin, Germany \\ ${ }^{\mathrm{b}}$ Institute for Physics of Microstructures of Russian Academy of Sciences, \\ Nizhny Novgorod, Russia
}

\begin{abstract}
Mo/Be multilayers are promising optical elements for extreme ultraviolet (EUV) lithography and space optics. Experimentally derived optical constants are necessary for accurate and reliable design of beryllium-containing optical coatings. We report optical constants of beryllium derived from synchrotron radiation-based reflectivity data of $\mathrm{Mo} / \mathrm{Be}$ multilayers. Results are in good agreement with available data in the literature obtained from the well-known absorption measurements of beryllium thin films or foils. We demonstrate synchrotron based at-wavelength reflectometry as an accurate and non-destructive technique for deriving EUV optical constants for materials that are difficult or unstable to make thin foils for absorption measurements. () The Authors. Published by SPIE under a Creative Commons Attribution 4.0 Unported License. Distribution or reproduction of this work in whole or in part requires full attribution of the original publication, including its DOI. [DOI: 10.1117/1.OE.60.4.044103]
\end{abstract}

Keywords: optical constants; extreme ultraviolet multilayers optics; molybdenum; beryllium; reflectometry; synchrotron radiation.

Paper 20201213 received Oct. 14, 2020; accepted for publication Mar. 10, 2021; published online Apr. 10, 2021.

\section{Introduction}

Beryllium is an essential material for varieties of scientific and industrial applications, due to its unique physical and optical properties. ${ }^{1,2}$ Beryllium in the form of oxide ceramics, metallic alloys, and salts is used in various industrial sectors. ${ }^{3}$ The fact that it has a low mass absorption coefficient in the x-ray range qualifies it for $\mathrm{x}$-ray windows, refractive lenses, and detector windows for synchrotron- and FEL-beamlines. ${ }^{4}$ Moreover, beryllium has a relatively low absorption near its $K$-edge compared to other lightweight materials such as $\mathrm{Si}, \mathrm{B}_{4} \mathrm{C}$, and $\mathrm{SiC}$. This low absorption property makes beryllium a promising candidate material for applications in the spectral range 30 to $180 \mathrm{eV}$, which constitutes extreme ultraviolet (EUV) regime. One application in this range for beryllium is as a bandpass filter in EUV astronomy instruments, ${ }^{5}$ the low absorption around $13.4 \mathrm{~nm}(\sim 92.5 \mathrm{eV})$ makes beryllium an attractive material in the technological development of EUV lithography optics. Most of the benefits of beryllium in the EUV range comes from its low absorptive nature. The absorption coefficients in the EUV range is indeed lower than other traditional low- $Z$ materials such as $\mathrm{B}_{4} \mathrm{C}, \mathrm{SiC}$, and $\mathrm{Si}$ as shown in Fig. 1(a). The dispersive component of the refractive index of beryllium is also comparable as given in Fig. 1(b).

Calculations of certain beryllium-containing new multilayer designs, using optical constants of beryllium from the Henke table ${ }^{7}$ achieve reflectivity higher than $70 \%$ in the 11- to 13.4-nm spectral range. ${ }^{7}$ This range is of high interest for EUV lithography applications. ${ }^{8,9}$ Berylliumcontaining multilayers also demonstrate high reflectivity at 17.1- and 30.4-nm wavelengths that are of interest for the optical engineering of solar mission satellites..$^{10}$ It is, therefore, indispensable to study optical constants of beryllium in the wider range of EUV targeting various

*Address all correspondence to Mewael Giday Sertsu, mewael.sertsu@helmholtz-berlin.de 


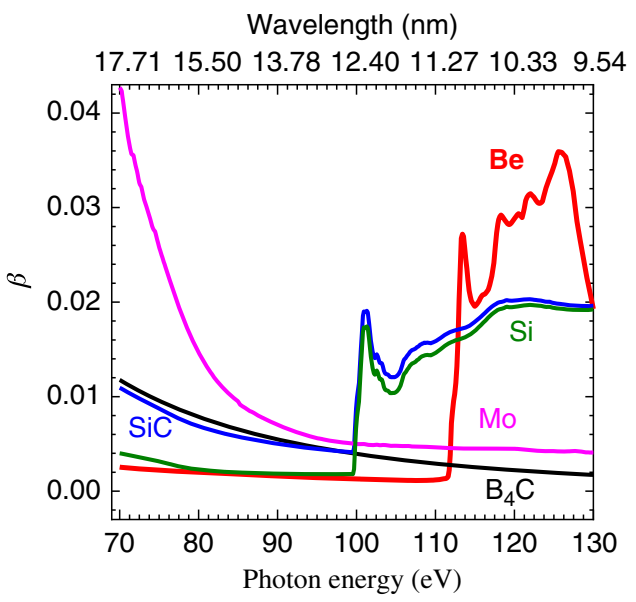

(a)

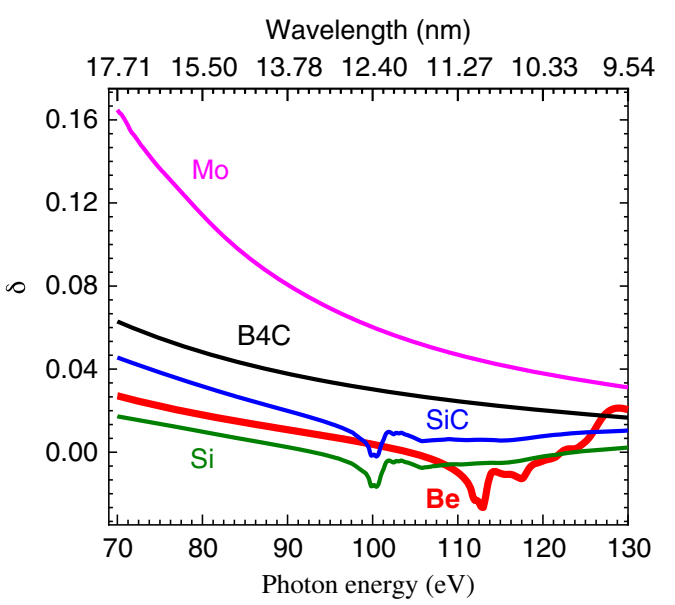

(b)

Fig. 1 Optical constants of bulk beryllium in comparison to the commonly used low- $Z$ materials $\mathrm{B}_{4} \mathrm{C}, \mathrm{SiC}$, and Si. (a) Absorption constants and (b) refractive optical constants. ${ }^{6}$

applications. However, it is particularly important to study optical constants near its $\mathrm{K}$ edge $(\sim 11.09 \mathrm{~nm}$ or $\sim 111.7 \mathrm{eV})$ where optical responses show abrupt changes due to extremely sensitive interactions of fine structures with neighboring absorbing atoms. It is convenient to discuss optical constants in EUV and x-ray spectra in terms of $\delta$ and $\beta$ to which the refractive index $\mathrm{n}$ is related as

$$
n=1-\delta \pm i \beta,
$$

where $1-\beta$ is the real part (i.e., dispersive) and $\beta$ is the imaginary (absorptive) part of the refractive index. Apart from the reports in Ref. 6 and in our recent work, ${ }^{11}$ measured optical constants of beryllium are limited in the literature. We focus on the measurement of optical constants near the beryllium absorption edge, where one can expect that experimentally derived optical constants to show strongest deviation from the calculated constants based on atomic scattering factors from Henke tables.

In this context, a combined analysis of x-ray and EUV reflectometry measurements has been reported in recent years. Reconstruction of optical and interface parameters of $\mathrm{Mo} / \mathrm{Si}$ and $\mathrm{B}_{4} \mathrm{C} / \mathrm{CeO}_{2}$ MLs from grazing incidence EUV reflectivity measurements using thicknesses from independent XRR data analysis are reported in Refs. 12-14. Simultaneous analysis of normal incidence EUV reflectivity and XRR data were reported in Refs. 15-17 for the characterization of La/B multilayers. Similar methodologies but technically improved are applied in this work to derive optical constants of beryllium around its $K$-edge with high accuracy and reliability. The XRR measurements at $\mathrm{Cu}-\mathrm{K} \alpha(\sim 8 \mathrm{keV})$ enables determination of ML thicknesses (period, layer, and interlayer thicknesses) with high spatial resolution. The EUV measurements determine optical constants with high sensitivity to optical fluctuations.

In this paper, at-wavelength grazing incidence EUV reflectivity measurements are performed to derive optical constants of beryllium. The method takes into consideration the sensitivity of optical constants to the configuration of an atom in its environment (i.e., resolution of fine structures). ${ }^{12}$ The method is further optimized by taking high-resolution energy measurements to account for abrupt changes in optical responses especially near the beryllium $\mathrm{K}$ edge. Wideangle reflectivity measurements are carried out to collect at least two Bragg peaks that allow contributions of all layers and interlayers in the ML stack to be determined. By choosing a robust numerical algorithm to fit the measured data [genetic algorithm (GA) in this case], the combined analysis results in accurate and reliable optical constants. To our knowledge, this method is the only alternative to derive EUV and soft x-ray optical constants from materials that are difficult to produce as freestanding foils (e.g., $\mathrm{Mg}$ ), which are important for space mission optics. 


\section{Sample Description, Experiments, and Data Analysis}

As explained in the introduction, experiments were designed in such a way that structural and optical parameters of MLs are derived with adequate reliability and accuracy. Two different multilayer structures were designed and fabricated on Si-substrates using DC magnetron sputtering facilities that are certified according to health and safety standards ${ }^{10}$ and pumped out to a residual pressure of $4 \times 10^{-5} \mathrm{~Pa}$. The working pressure of Argon (Ar) during the deposition was about $0.1 \mathrm{~Pa}$, and the chemical purity of Ar was about $99.99 \%$. The target materials were disks of diameter $150 \mathrm{~mm}$ and thickness $5 \mathrm{~mm}$. The process utilized $270 \mathrm{~W}$ of power for the Be target, $160 \mathrm{~W}$ for the Mo target, and $150 \mathrm{~W}$ for the Si target.

The first sample (sample_01) is a tri-layer structure of [Mo/Be/Si] on Si substrate with design period $d \sim 7 \mathrm{~nm}\left(d_{\mathrm{Si}}=2.6 \mathrm{~nm}, d_{\mathrm{Be}}=2 \mathrm{~nm}\right.$, and $\left.d_{\mathrm{Mo}}=2.4 \mathrm{~nm}\right)$ and number of tri-layers $N=110$. Sample_01 was designed to demonstrate high reflectivity performance at the wavelength of interest for EUV lithography, i.e., around $13.5 \mathrm{~nm}$. Introducing Si into the [Mo/Be] MLM design plays a smoothing effect of interfaces and thus enhancing reflectivity. ${ }^{6}$

The second sample (sample_02) was [Mo/Be] on Si substrate with design parameters of period $d=14 \mathrm{~nm}, \Gamma=0.36, d_{\mathrm{Mo}}=5.04 \mathrm{~nm}, d_{\mathrm{Be}}=8.96 \mathrm{~nm}$, and $N=25$. The $\Gamma$-ratio is a term defined as a quotient of thickness of the more absorbing layer by virtue of its mass (hence absorber layer) to period. In [Mo/Be] ML combination, Mo with mass density of $10.28 \mathrm{gm} / \mathrm{cm}^{3}$ is an absorber layer. Sample_02 is designed to allow at-wavelength EUV Reflectivity (EUVR) measurements with the intention of obtaining at least one Bragg peak in a $\theta$ to $2 \theta$ scan. Since the purpose of sample_01 is just to test EUV-reflectivity performance, only spectral measurements were performed around the working wavelength in normal incidence. On the other hand, sample_02 was measured using at-wavelength grazing incidence EUV reflectivity from 90 to $134 \mathrm{eV}$ with an energy step $E_{\text {step }}=0.5 \mathrm{eV}$ in the 90 to $110 \mathrm{eV}$ range, $0.2 \mathrm{eV}$ in the 110 to $116 \mathrm{eV}$ range around the K-edge of beryllium, and $1 \mathrm{eV}$ in 116 to $134 \mathrm{eV}$ range. All EUV measurements for both samples were performed in the reflectometer end station of the optics beamline at the BESSY-II synchrotron radiation source at an energy resolution in the order of few $\mathrm{meV}^{18,19}$ In addition, complementary x-ray reflectivity (XRR) measurements at a photon energy of $8.048 \mathrm{keV}(\mathrm{Cu} \mathrm{K} \alpha)$ were performed using a Philips X'Pert Pro diffractometer system, with a high-resolution asymmetric four-crystal Ge (220) monochromator, at the Institute for Physics of Microstructures of Russian Academy of Sciences (IPM-RAS).

Reconstruction of ML parameters from both XRR and EUVR data was performed with the help of IMD program (modeling and analysis of multilayer films) where details of the mathematical formulations are provided in Ref. 20. Computations of optical functions (reflection and transmission) of multilayer films in IMD is a three-step process. First, reflections/transmissions are calculated using Fresnel equations at each optical interfaces. Then computation of electromagnetic plane waves in each layer by solving Maxwell's equations. Finally, using a recursive approach to compute the net field amplitude throughout the stack, starting at the bottommost layer.

The numerical fitting in IMD benefits from robust mathematical algorithms. GA and a more complex variant known by differential evolution (DE), which were included in the version 5 of IMD package, are used for the fittings in this report. GA is considered as a global optimization algorithm as it is generally less sensitive to the choice of initial parameters, less susceptible to local minima, and undergoes stochastic search of global minima in a parameter space with an intelligent strategy of solution finding. ${ }^{21,22}$ A nonlinear curve fitting of the measured reflectivity data against a goodness of fit parameter chi-square $(\chi 2)$ similar to the Pearson's criterion ${ }^{20}$ retrieves almost any parameter of the ML. However, a realistic ML modeling is required to perform the nonlinear fitting. Thus, the ML structure of sample_02 is modeled in a four-layer system (i.e., layer $1+$ interlayer_1 + layer 2 + interlayer_2) to account inter-diffusion regions as independent layers as witnessed by the high resolution TEM image (Fig. 2) of a similar sample that was deposited in the same facility with very similar deposition conditions as sample_02. The TEM image provides qualitative confirmation for the formation of interlayers due diffusions and substantiate the adoption of the four-layer model. The fitting is a two-step process. First, thicknesses and roughness are derived from x-ray measurement at $8.048 \mathrm{keV}(0.15 \mathrm{~nm})$ benefiting from high spatial depth resolution. Results from the XRR fitting are transferred to the second 


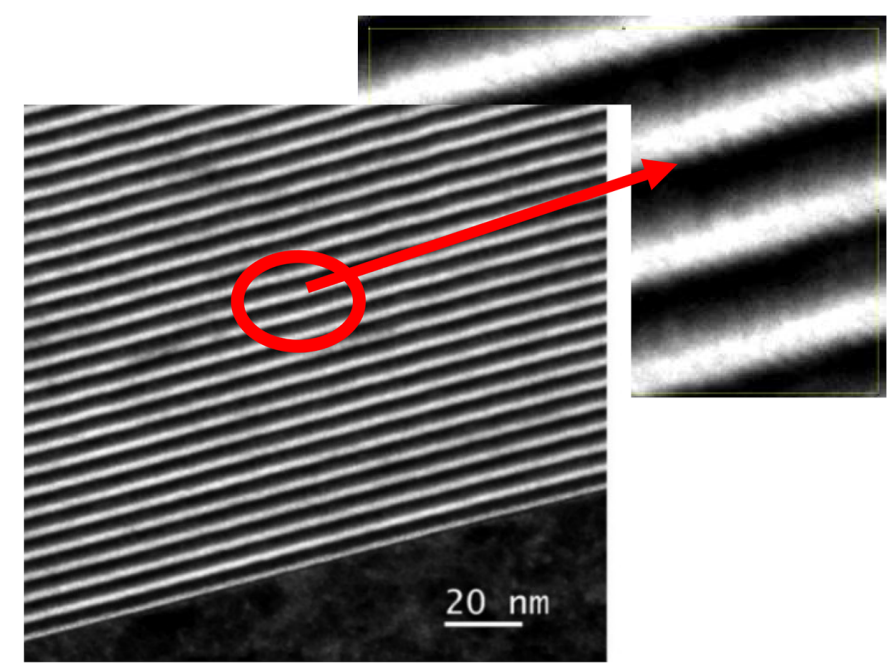

Fig. 2 High-resolution bright field transmission electron microscopy image of Mo/Be sample to demonstrate formation of interlayers at interfaces. The ML sample was deposited in the same sputtering machine under similar deposition conditions to the samples discussed in this work.

step fitting in EUV to retrieve optical constants $(\delta$ and $\beta$ ) of each layer and interlayer. In the fourlayer model, fixing the thicknesses and roughness lowers to at least eight input parameters, $\delta$ and $\beta$ of each layers and interlayers, excluding the surface and substrate parameters.

\section{Results and Discussion}

High measured reflectivity performance of $70.42 \%$ at $13.42-\mathrm{nm}$ EUV wavelength in normal incidence is achieved by the tri-layer structure of sample_01 as shown in Fig. 3(a). Figure 3(b) shows angular measurements of that sample at a wavelength of $13.37 \mathrm{~nm}$. Average thicknesses of $d_{\mathrm{Si}}=3.09 \mathrm{~nm}, d_{\mathrm{Be}}=1.07 \mathrm{~nm}, d_{\mathrm{Mo}}=2.70 \mathrm{~nm}$ are obtained from X-ray reflectometry (XRR) data analysis. The incorporation of Si layers into the $\mathrm{Mo} / \mathrm{Be}$ structures enhances performance as intensively reported in Ref. 23.

A nonlinear fit of the XRR of the four-layer Mo/Be structure (Sample_02) is shown in Fig. 4(a). The model structure and derived layer and interlayer thicknesses are given in Fig. 4(b).

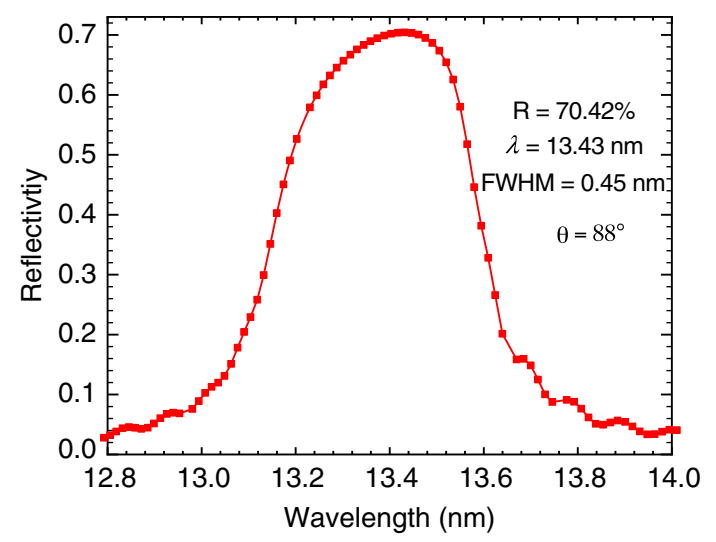

(a)

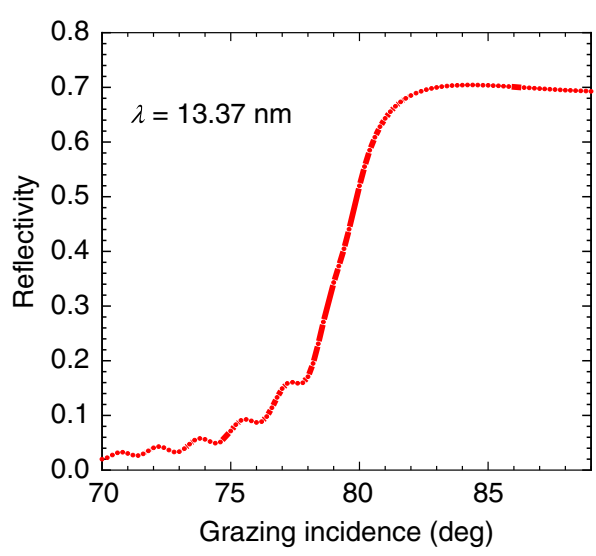

(b)

Fig. 3 Measured reflectivity of the $[\mathrm{Mo} / \mathrm{Be} / \mathrm{Si}] \times 110$ multilayer (sample_01) at EUV wavelengths. (a) Spectral dependence in near-normal incidence and (b) angular dependence at $13.37 \mathrm{~nm}$. 


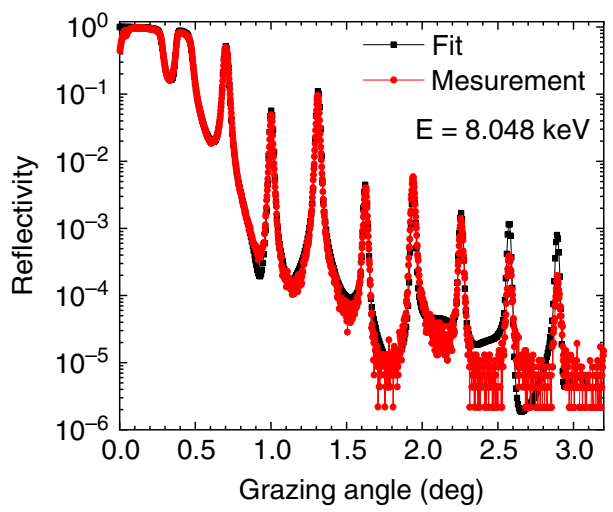

(a)

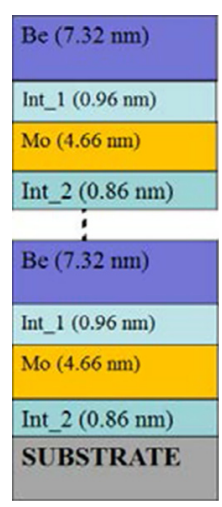

(b)

Fig. 4 (a) Measured and fitted XRR for sample_02 and (b) the sketch of the four-layer model implemented for fitting the x-ray data. Bulk densities are used for fitting.

The analysis of the XRR measurement at $\mathrm{Cu}-\mathrm{K}_{\alpha}$ line returns a period $d=13.8 \pm 0.2 \mathrm{~nm}$, $d_{\mathrm{Mo}}=4.66 \pm 0.2 \mathrm{~nm}$, and $d_{\mathrm{Be}}=7.32 \pm 0.2 \mathrm{~nm}$. In addition, the fitting resulted in asymmetric thicknesses for the interlayers with $d_{\text {Mo-on-Be }}=0.8 \pm 0.2 \mathrm{~nm}$ and $d_{\text {Be-on-Mo }}=0.96 \pm 0.2 \mathrm{~nm}$. Optical constants $(\delta$ and $\beta$ ) are derived from EUVR measurements performed at each photon energy by adopting the model and thicknesses obtained from the XRR fit, shown in Fig. 4(b). This approach has an advantage in minimizing the number of input parameters. Examples of fits to the measured curves at 99.61 and $121.64 \mathrm{eV}$ are given in Fig. 5.

In this way, the EUVR fitting solely fits the optical constants $(\delta$ and $\beta)$ by taking into account the thickness parameters adopted from the XRR analysis. This method has two major advantages: in one hand, it minimizes the number of input parameters significantly, second, it increases the accuracy of the analysis due to the high optical sensitivity in this regime of the spectrum. Yakunin et al. ${ }^{24}$ have already reported the advantage of simultaneous analysis of EUV and X-ray data in driving structural parameters of multilayers, the mass density, and thicknesses. For the derivation of optical constants in EUV, however, systematic analysis as implemented in this work, can give better results. Derived average optical constants of beryllium are summarized in Fig. 6 based on the methodology discussed above. The derived optical constants of beryllium show very good agreement with Soufli et al. ${ }^{6}$ in the Henke table and to recently published work in Ref. 11.

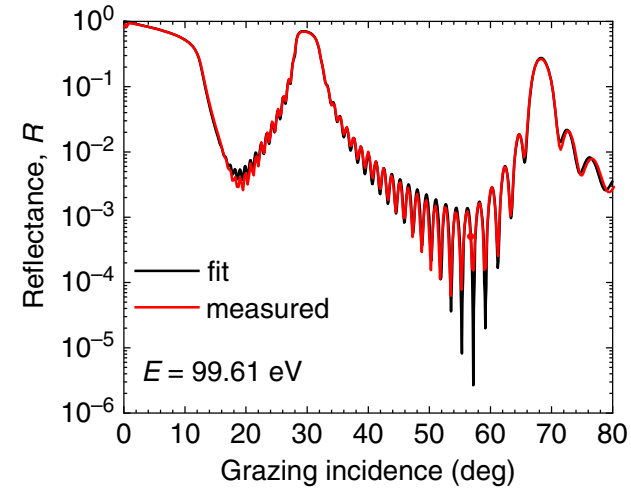

(a)

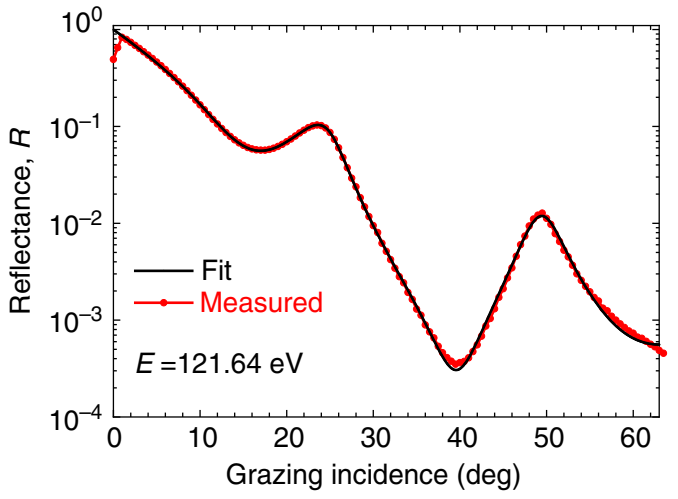

(b)

Fig. 5 EUV measurements and corresponding fits of sample_02 (a) at $99.61 \mathrm{eV}$ and fit, (b) at the $121.64 \mathrm{eV}$. The layer and interlayer thicknesses are taken from the results of the XRR data in Fig. 4. 


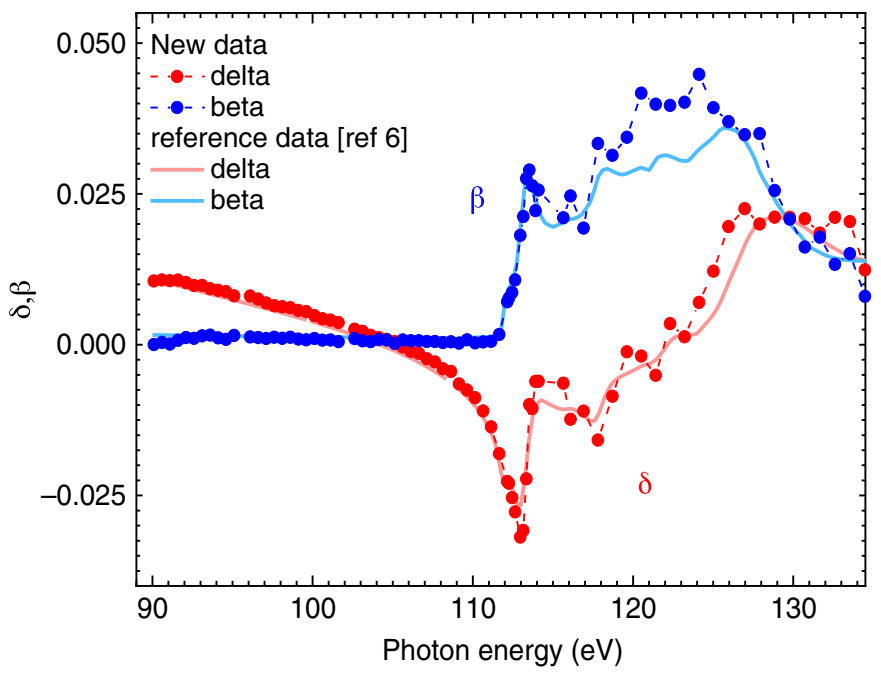

Fig. 6 Derived optical constants of beryllium in the energy range 90 to $134 \mathrm{eV}$ from $[\mathrm{Mo} / \mathrm{Be}] \times$ $25 \mathrm{ML}$ structure (sample_02) by combining at-wavelength reflectometry in x-ray and EUV energy range (circles). Solid lines are reference data from Ref. 7.

\section{Conclusion}

We have presented an alternative, non-destructive, realistic, and simpler method for deriving beryllium optical constants by combining XRR and EUVR measurements. The method is suitable for instable or reactive materials, which cannot be produced as thin films for absorption measurements. The method has been tested on $\mathrm{Mo} / \mathrm{Be}$ multilayers in the range of the $\mathrm{Be} K$-edge at which beryllium has high technological importance for EUV-lithography optics as potential spacer material, since beryllium-containing multilayer coatings show high reflectivity performance. We report reflectivity larger than $70 \%$ at $13.4 \mathrm{~nm}$ from $\mathrm{Mo} / \mathrm{Be} / \mathrm{Si}$ multilayers. The introduction of beryllium into the $\mathrm{Mo} / \mathrm{Si}$ structures enhances performance. Optimization of the coating processes in magneton sputtering may boost the reflectivity performance further. The optical constants of beryllium derived by our method demonstrate good agreement with available literature data obtained by absorption measurements. The derived optical constants help for accurate and reliable design of beryllium-containing coatings.

\section{Acknowledgments}

We would like to acknowledge Deutsche Forschungsgemeinschaft (DFG) (SO 282 1497/1-1 and the samples fabrication and laboratory structural characterization were carried out in the framework of Russian State Assignment No. 0030-2021-0022.

\section{References}

1. A. T. Marshall, D. Carde, and M. Kent, "Improved vacuum evaporation unit for beryllium coating for biological x-ray-microanalysis," J. Microsc.-Oxford 139, 335-337 (1985).

2. A. T. Marshall and D. Carde, "Beryllium coating for biological x-ray-microanalysis," J. Microsc.-Oxford 134, 113-116 (1984).

3. T. P. Taylor et al., "Beryllium in the environment: a review," J. Environ. Sci. Health Part A 38(2), 439-469 (2003).

4. J. J. Truhan and L. M. Wagner, "Fabricating thin beryllium windows for x-ray applications," Nucl. Instrum. Methods 176(3), 481-485 (1980).

5. B. J. Kent et al., "EUV band-pass filters for the ROSAT wide field camera," Proc. SPIE 1344 (1990).

6. R. Soufli, S. Bajt, and E. M. Gullikson, "Optical constants of beryllium from photoabsorption measurements for x-ray optics applications," Proc. SPIE 3767, 251-258 (1999). 
7. Y. A. Vainer et al., "Beryllium-based multilayer mirrors for the soft x-ray and extreme ultraviolet wavelength ranges," J. Surf. Investigation 14, 124-134 (2020).

8. S. Bajt, "Molybdenum-ruthenium/beryllium multilayer coatings," J. Vacuum Sci. Technol. A Films 18(2), 557-559 (2000).

9. J. A. Folta et al., "Advances in multilayer reflective coatings for extreme ultraviolet lithography," Proc. SPIE 3676, 702-709 (1999).

10. N. I. Chkhalo et al., "Be/Al-based multilayer mirrors with improved reflection and spectral selectivity for solar astronomy above 17 nm wavelength," Thin Solid Films 631, 106-111 (2017).

11. N. C. M. Svechnikov et al., "Optical constants of sputtered beryllium thin films determined from photoabsorption measurements in the spectral range 20.4-250 eV," J. Synchrotron. Radiat. 27(Pt. 1), 75-82 (2019).

12. M. G. Sertsu et al., "Analysis of buried interfaces in multilayer mirrors using grazing incidence extreme ultraviolet reflectometry near resonance edges," Appl. Opt. 54(35), 10351-10358 (2015).

13. M. G. Sertsu et al., "Deposition and characterization of $\mathrm{B}_{4} \mathrm{C} / \mathrm{CeO}_{2}$ multilayers at $6 . \times \mathrm{nm}$ extreme ultraviolet wavelengths," J. Appl. Phys. 119(9), 095301 (2016).

14. A. Comisso et al., "Characterization of $\mathrm{TiO}_{2}$ thin films in the EUV and soft x-ray region," Proc. SPIE 9510, 95100Z (2015).

15. I. A. Makhotkin et al., "Short period $\mathrm{La} / \mathrm{B}$ and $\mathrm{LaN} / \mathrm{B}$ multilayer mirrors for similar to 6.8 nm wavelength," Opt. Express 21(24), 29894-29904 (2013).

16. I. A. Makhotkin et al., "Spectral properties of $\mathrm{La} / \mathrm{B}$ - based multilayer mirrors near the boron K absorption edge," Opt. Express 20(11), 11778-11786 (2012).

17. I. A. Makhotkin et al., "Wavelength selection for multilayer coatings for lithography generation beyond extreme ultraviolet," J. Micro-Nanolithogr. MEMS MOEMS 11(4), 040501 (2012).

18. A. Sokolov et al., "At-wavelength metrology facility for soft x-ray reflection optics," Rev. Sci. Instrum. 87(5), 052005 (2016).

19. F. Schafers et al., "The at-wavelength metrology facility for UV- and XUV-reflection and diffraction optics at BESSY-II," J. Synchrotron. Radiat. 23, 67-77 (2016).

20. D. L. Windt, "IMD—software for modeling the optical properties of multilayer films," Comput. Phys. 12(4), 360-370 (1998).

21. P. D. Binda and F. E. Zocchi, "Genetic algorithm optimization of x-ray multilayer coatings," Proc. SPIE 5536, 97-108 (2004).

22. A. Ulyanenkov, K. Omote, and J. Harada, "The genetic algorithm: refinement of x-ray reflectivity data from multilayers and thin films," Physica B 283(1-3), 237-241 (2000).

23. N. I. Chkhalo et al., "High-reflection Mo/Be/Si multilayers for EUV lithography," Opt. Lett. 42(24), 5070-5073 (2017).

24. S. N. Yakunin et al., "Combined EUV reflectance and x-ray reflectivity data analysis of periodic multilayer structures," Opt. Express 22(17), 20076-20086 (2014).

Mewael Giday Sertsu is a physicist working as a beamline scientist and postdoc researcher in the Department of Optics and Beamlines at the Helmholtz-Zentrum Berlin (HZB) since 2017. Prior to joining to the $\mathrm{HZB}$, he was a $\mathrm{PhD}$ student and Erasmus Mundus scholar at Padova University (Italy) and RWTH Aachen University (Germany). His research interests are in the areas of nanometer optics, EUV and x-ray sciences, EUV metrology, thin film, and multilayer coatings.

Andrey Sokolov is an X-ray optics physicist working in the Department of Optics and Beamlines at BESSY II synchrotron facility of HZB für Materialien und Energie since 2013. His main research area is connected to precision at-wavelength metrology on XUV optics, developments of design and conceptions for new XUV optical elements, and design for XUV spectrometers and beamlines. Previously, he worked at Saint Petersburg State University in Solid State Electronics Department, where he was actively involved in research on investigation of thin layered system internal structure and interlayers by $\mathrm{x}$-ray spectroscopic techniques.

Nikolay Chkhalo is an $\mathrm{x}$-ray optics physicist and technologist. His main research interests are reflective $\mathrm{x}$-ray optics, fabrication and metrology of supersmooth and ultrahigh-precision 
surfaces for diffraction-limited x-ray and EUV optics, deposition and metrology of thin coatings. Since 2013 he is the head of Multilayer x-ray optics department of IPM RAS.

Vladimir Polkovnikov is an x-ray optics physicist and technologist, senior researcher, and head of laboratory of IPM RAS. He has worked in IPM since 2002, and his works mainly concern the technology of high-vacuum deposition of highly stable, effective and precise x-ray and EUV coatings for optical application, including space telescopes, x-ray microscopy, EUV lithography.

Nikolay Salashchenko is an X-ray optics physicist, who started investigation and development of x-ray coatings in IPM RAS. His main research interests are optimization and deposition of reflective and x-ray optics and free-standing absorption filters, physical processes in ultrathin metal layers, development of technologies for creation x-ray optical elements. He is a corresponding member of the RAS since 2000 and head of the Multilayer X-ray Optics Department of IPM RAS from 1993 to 2013.

Mikhail Svechnikov is a postdoc researcher, working in IPM RAS since 2012, where he obtained a $\mathrm{PhD}$. He is working on $\mathrm{x}$-ray modeling and diagnostics of multilayer structures, and his main research interests are modelling of reflectivity and elastic scattering in non-ideal multilayer films and solution of inverse problem of such modeling.

Franz Schäfers is a senior scientist (physicist) in the Department of Optics and Beamlines at BESSY II of HZB für Materielien und Energie. Some of his research interests include soft x-ray optics and technology, multilayers, reflectometry and hard x-ray photoelectron spectroscopy. 